\title{
CO luminosity - line-width correlation of submillimeter galaxies and a possible cosmological application (Research Note)
}

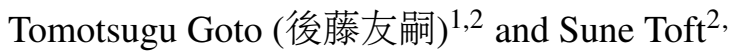

\author{
1 National Tsing Hua University, No. 101, Section 2, Kuang-Fu Road, Hsinchu, Taiwan 30013, ROC \\ e-mail: tomo@phys.nthu.edu.tw \\ 2 Dark Cosmology Centre, Niels Bohr Institute, University of Copenhagen, Denmark \\ e-mail: sune@dark-cosmology.dk
}

Received 10 March 2015 / Accepted 21 April 2015

\section{ABSTRACT}

\begin{abstract}
Context. A possible correlation between CO luminosity $\left(L_{\mathrm{CO}}^{\prime}\right)$ and its line width (FWHM) has been suggested and denied in the literature. Such claims were often based on a small or heterogeneous sample of galaxies, hence were inconclusive. Aims. We aim to prove or disprove the $L_{\mathrm{CO}}^{\prime}$-FWHM correlation.

Methods. We compiled a large sample of sub-mm galaxies at $z>2$ from the literature and investigated the $L^{\prime} \mathrm{CO}-\mathrm{FWHM}$ relation. Results. After carefully evaluating the selection effects and uncertainties, such as inclination and magnification via gravitational lensing, we show that a weak but significant correlation exists between $L_{\mathrm{CO}}^{\prime}$ and FWHM. We also discuss a feasibility to measure the cosmological distance using the correlation.
\end{abstract}

Key words. methods: observational - distance scale - submillimeter: galaxies

\section{Introduction}

A possible correlation between $\mathrm{CO}$ luminosity $\left(L_{\mathrm{CO}}^{\prime}\right)$ and its line width at high $z$ has been suggested in the literature. Bothwell et al. (2013) observed 32 sub-mm galaxies (SMG) at $1.2<z<$ 4.1 in ${ }^{12} \mathrm{CO}$ and suggested a correlation between $\mathrm{CO}$ luminosity and line width (FWHM) in high-redshift starbursts, probably relating to baryon-dominated gas dynamics within the regions probed (see their Fig. 5). Using $15 \mathrm{SMG}$ at $z=2-4$ with known lens magnifications, Harris et al. (2012) show that the SMGs fall close to a power law with small scatter in the CO luminosity and line width (FWHM) plane (see their Fig. 7).

Bright SMGs are often gravitationally-lensed. Such relation between $L_{\mathrm{CO}}^{\prime}$ and FWHM can be used to estimate magnification factor $(\mu)$. Indeed, Harris et al. (2012) used the relation to estimate luminosity of SMGs with unknown $\mu$. Fu et al. (2013) also used this relation to estimate a small $\mu(\sim 1.8)$ of merging galaxies at $z=2.3$ to conclude that their star formation rate was $2000 M_{\odot} \mathrm{yr}^{-1}$ (see their Fig. S7). Similarly, Riechers et al. (2013) used the relation to estimate $\mu(1.5 \pm 0.7)$ for a starburst galaxy at $z=6.34$, finding its SFR of $2900 M_{\odot} \mathrm{yr}^{-1}$.

Conversely, Carilli \& Walter (2013) examined the relation using a much larger sample and found no significant correlation between $L_{\mathrm{CO}}^{\prime}$ and FWHM (see their Fig. 5). However, their sample was heterogeneous because it included various types of galaxies, such as SMM, QSO, radio galaxies, and $24 \mu \mathrm{m}$ sources. Therefore, the existence of $L_{\mathrm{CO}}^{\prime}$-FWHM correlation is still controversial.

In this paper, we compile a larger sample of $z>2$ galaxies with $\mathrm{CO}$ detection from the literature, to re-examine the $L_{\mathrm{CO}}^{\prime}$-FWHM correlation at high $z$. We carefully examine various sources of possible uncertainties, such as different types of galaxies, luminosity conversion from different $\mathrm{CO}$ transitions, and errors in estimating $\mu$. Unless otherwise stated, we adopt a cosmology with $\left(h, \Omega_{\mathrm{m}}, \Omega_{\Lambda}\right)=(0.7,0.3,0.7)$.

\section{Sample}

Our sample is based on the updated version of the supplemental table of Carilli \& Walter (2013), which contains all published CO line measurements at $z>2$ as of December 31, 2012. The updated version also includes some sub-mm sources published in 2013. We created a large sample of high- $z$ sub-mm galaxies by adding 32 sources observed by Bothwell et al. (2013). In total, our sample contains 195 galaxies that were detected at least in one $\mathrm{CO}$ transition, with a measured FWHM.

\section{Analysis}

We compute $L_{\mathrm{CO}}^{\prime}$ using the standard definition

$L_{\mathrm{CO}}^{\prime}=3.25 \times 10^{7} S_{\mathrm{CO}} \Delta v v_{\mathrm{obs}}^{-2} D_{\mathrm{L}}^{2}(1+z)^{-3}$,

where $S_{\mathrm{CO}} \Delta v$ is the velocity integrated line flux, $L_{\mathrm{CO}}^{\prime}$ is the line luminosity in $\mathrm{K} \mathrm{Km} \mathrm{s}^{-1} \mathrm{pc}^{2}, v_{\mathrm{obs}}$ is the observed central frequency of the line, and $D_{\mathrm{L}}$ the luminosity distance in Mpc.

If the molecular gas emission comes from thermalized, optically thick regions, $L_{\mathrm{CO}}^{\prime}$ is constant for all $J$ levels. However, in reality, the ratio of $L_{\mathrm{CO}}^{\prime}(1-0)$ to higher order $L_{C 0}^{\prime}(J, J-1)$ depends on temperature and density. We use average ratio of $L_{\mathrm{CO}}^{\prime}(J, J-1) / L_{\mathrm{CO}}^{\prime}(1-0)$, provided in Table 4 of Bothwell et al. (2013), to calculate $L_{\mathrm{CO}}^{\prime}(1-0)$ from higher order CO observations. Higher redshift galaxies are often observed only at mid-tohigh- $J$ CO transitions. We discuss uncertainties associated with this conversion in Sect. 4 in detail. 
Table 1. Correlation between $L_{\mathrm{CO}}^{\prime} / \mu$ and FWHM for various samples.

\begin{tabular}{cccllll}
\hline \hline Sample & $N$ & Spearman's coeff. & $p$-value & Kendall's coeff. & $p$-value & Dispersion $\left(\mathrm{km} \mathrm{s}^{-1}\right)$ \\
\hline All & 195 & 0.55 & $1.7 \times 10^{-16}$ & 0.39 & $<1.0 \times 10^{-16}$ & 187. \\
\hline Submm galaxies & 72 & 0.53 & $2.1 \times 10^{-6}$ & 0.37 & $4.2 \times 10^{-6}$ & 272. \\
QSO hosts & 31 & 0.53 & $2.0 \times 10^{-3}$ & 0.39 & $1.9 \times 10^{-3}$ & 245. \\
Submm galaxies +QSO hosts & 103 & 0.52 & $1.4 \times 10^{-8}$ & 0.37 & $<1.0 \times 10^{-16}$ & 218. \\
\hline CO(4-3) only & 26 & -0.34 & $9.2 \times 10^{-2}$ & -0.21 & $1.4 \times 10^{-1}$ & 279. \\
CO(3-2) only & 62 & 0.12 & $3.5 \times 10^{-1}$ & 0.09 & $2.8 \times 10^{-1}$ & 217. \\
CO(2-1) only & 38 & 0.18 & $2.8 \times 10^{-1}$ & 0.14 & $2.2 \times 10^{-1}$ & 182. \\
CO(1-0) only & 43 & 0.22 & $1.5 \times 10^{-1}$ & 0.15 & $1.6 \times 10^{-1}$ & 121. \\
\hline All but CO(4-3) & 143 & 0.52 & $4.0 \times 10^{-11}$ & 0.37 & $<1.0 \times 10^{-16}$ & 206. \\
All but CO(3-2) & 107 & 0.56 & $2.5 \times 10^{-10}$ & 0.40 & $<1.0 \times 10^{-16}$ & 201. \\
All but CO(2-1) & 131 & 0.58 & $5.9 \times 10^{-13}$ & 0.42 & $<1.0 \times 10^{-16}$ & 231. \\
All but CO(1-0) & 126 & 0.52 & $4.8 \times 10^{-10}$ & 0.37 & $<1.0 \times 10^{-16}$ & 237. \\
\hline$\mu$ known & 85 & 0.42 & $7.3 \times 10^{-5}$ & 0.30 & $5.3 \times 10^{-5}$ & 156. \\
$\mu$ unknown & 110 & 0.47 & $2.4 \times 10^{-7}$ & 0.33 & $3.6 \times 10^{-7}$ & 205. \\
\hline $24 \mu$ m sources & 8 & 0.45 & $2.6 \times 10^{-1}$ & 0.36 & $2.2 \times 10^{-1}$ & 204. \\
BzK galaxies & 4 & 0.80 & $2.0 \times 10^{-1}$ & 0.67 & $1.7 \times 10^{-1}$ & 156. \\
\hline
\end{tabular}

Notes. Dispersion is around the best-fit power law. The $p$ value of $<0.05$ can be considered significant.

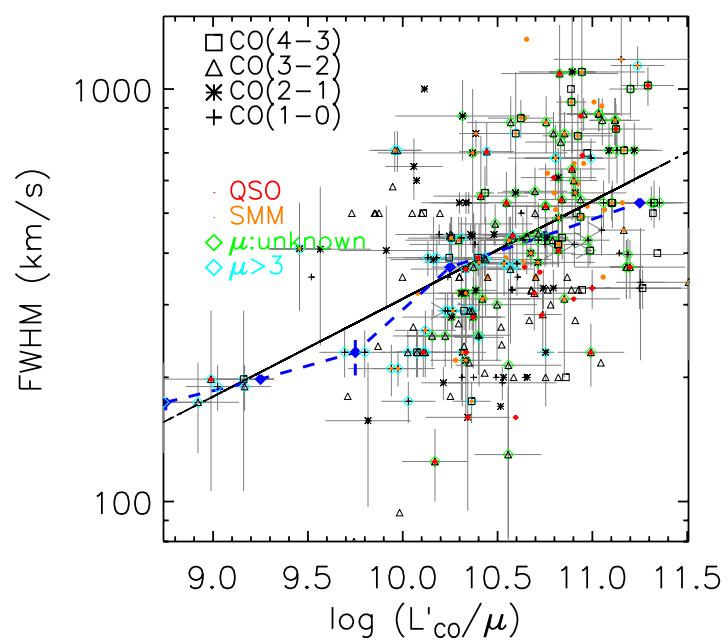

Fig. 1. Correlation between $L_{\mathrm{CO}}^{\prime} / \mu$ and FWHM for various samples. The blue dotted line connects the median in equally spaced logarithmic bins. The black solid line is the best-fit power law.

It is always a concern with high- $z$ objects that they might be gravitationally magnified. Submillimeter galaxies are not an exception, so $L_{\mathrm{CO}}^{\prime}$ also needs to be corrected for a magnification factor $(\mu)$, when the galaxy is magnified by a gravitational lens. We collected $\mu$ values from the literature whenever available. However, since $\mu$ must be estimated from detailed modeling of the lens potential, a reliable measurement is not always available. We examine how the correlation depends on the availability and amplitude of $\mu$ in Sect. 4, but to be brief, they do not change our main results. Most likely, majority of galaxies with missing $\mu$ are not strongly magnified; otherwise, a lens galaxy would have been detected and $\mu$ would have been estimated. We present $L_{\mathrm{CO}}^{\prime} / \mu$, whenever $\mu$ is available hereafter.

\section{Results}

\section{1. $L_{\mathrm{CO}}^{\prime} / \mu$-FWHM correlation}

In Fig. 1, we plot CO line width FWHM against $L_{\mathrm{CO}}^{\prime} / \mu$ for all galaxies in our sample. The solid line is the best-fit power law, which is

$$
\log (F W H M)=(0.24 \pm 0.03) \times \log \left(L_{\mathrm{CO}}^{\prime} / \mu\right)+(0.12 \pm 0.28)
$$

The significance of the non-zero slope is $8.7 \sigma$. Spearman's correlation coefficient is 0.55 with a $p$ value of $1.7 \times 10^{-16}$. Kendall's correlation coefficient is similarly significant as shown in Table 1. Errors on each point are small thanks to the large number of galaxies in our sample. The median values increase with increasing $L_{\mathrm{CO}}^{\prime} / \mu$. These results show that there is a weak, but very significant correlation between $L_{\mathrm{CO}}^{\prime} / \mu$ and FWHM.

The presence of such a correlation is not surprising, because $L_{\mathrm{CO}}^{\prime} / \mu$ is sensitive to the total mass of molecular gas, while the FWHM is sensitive to dynamical mass. The relation is similar to the Tully-Fisher relation, which is also a power law, in the local Universe (Tully \& Fisher 1977). A large scatter around the relation is also expected because of the inclination effect, along with the unknown $\mu$ for a subset of galaxies. We measured a dispersion around the best-fit line of $43 \%$, which is consistent with what one expects from randomly oriented thin disks (45\%), suggesting $\mu$ is close to 1 for most of the galaxies with unknown $\mu$.

\subsection{Uncertainty in converting different transitions}

We tested that conversions from different $\mathrm{CO}$ transitions can mimic the correlation. In Fig. 1, we used different symbols for different $\mathrm{CO}$ transitions $(4-3,3-2,2-1$, and 1-0). In Table 1 , we show the correlation coefficients for them. For $\mathrm{CO}(4-3)$, we do not see a positive correlation, while we see weak correlations for the other transitions. It is reassuring to see the correlations within single CO transitions, because they cannot be due to errors in the transition conversion. The reason we do not see a positive correlation for the $\mathrm{CO}(4-3)$ transition is not clear, but we point out that this is the smallest subsample and that since a single transition only spans a narrow redshift range (and thus, a narrow luminosity range), it is difficult to examine a correlation with a small sample in a single transition. It will be necessary to repeat this test using the wider frequency coverage of ALMA. On the other hand, we see significant correlations in all cases where we exclude a certain $\mathrm{CO}$ transition from the sample. These tests suggest that the $L_{\mathrm{CO}}^{\prime}$-FWHM correlation is not an artifact of $\mathrm{CO}$ order conversions. 


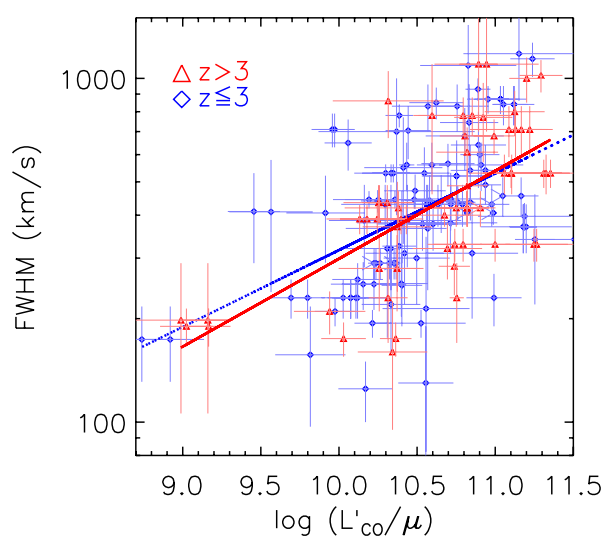

Fig. 2. $L_{\mathrm{CO}}^{\prime} / \mu$ plotted against FWHM. We separated the sample into $z>3$ (red triangles) and $z \leq 3$ (blue diamonds). The best-fit power laws for the two samples are FWHM $\left(\mathrm{km} \mathrm{s}^{-1}\right) \propto L_{\mathrm{CO}}^{\prime} / \mu^{0.22 \pm 0.04}(z \leq 3$, blue $)$ and FWHM $\left(\mathrm{km} \mathrm{s}^{-1}\right) \propto L_{\mathrm{CO}}^{\prime} / \mu^{0.25 \pm 0.04}(z>3$, red). The slopes are consistent with each other and with the slope derived from the full sample in Fig. 1.

\subsection{Dependence on magnification}

In our sample, 85 galaxies have known $\mu$, while the other 110 galaxies have not. We should first note that many sub-mm galaxies with unknown $\mu$ are probably not strongly magnified, or we would have seen the lens galaxy/cluster and measured $\mu$. With this in mind, we still test that an unknown $\mu$ can mimic the correlation. In Fig. 1, we show two extreme samples of galaxies, those with $\mu>3$ in cyan, and those with unknown $\mu$ in green. There is no apparent deviation from the best-fit relation, except that more $\mu$ s are unknown at higher redshift. In Table 1 , we separate the sample into those with known $\mu$ and those with unknown $\mu$, both of which show a significant correlation. Because brighter sub-mm galaxies are more likely to be lensed (owing to the steep luminosity function), the correction to unknown $\mu$ will steepen the correlation; i.e., the correlation will not disappear with the correction. It is reassuring to see a positive correlation using many sub-mm galaxies with unknown $\mu$.

\subsection{Dependence on galaxy types}

We also separate the sample according to galaxy types in Table 1 and Fig. 1. Unfortunately, $24 \mu \mathrm{m}$ sources and BzK galaxy samples are too small to examine the correlation. Both sub-mm galaxies (SMG) and QSOs have a significant correlation, with sub-mm galaxies having a greater significance.

\subsection{Redshift dependence}

Next we examine whether the $L_{\mathrm{CO}}^{\prime}$-FWHM correlation changes with redshift. Unfortunately, owing to the flux limits of the telescopes, the higher redshift sample includes more luminous sources, while lower redshift sources have smaller luminosity due to volume effects. With this limitation in mind, we separated the sample into high $(z>3)$ and low $(z \leq 3)$ redshift samples in Fig. 2. The best-fit lines to each sample agree with each other within the errors, which is consistent with no significant redshift evolution. The slopes are also consistent with each other and with that of the full sample in Fig. 1.

A concern for any low-level CO transition is that CMB temperature becomes higher at the highest redshift end. For example, the CMB temperature at $z=6$ is $\sim 19 \mathrm{~K}$, which is higher than the temperature needed to excite the $\mathrm{CO}(2-1)$ line $(16.6 \mathrm{~K})$. Fortunately at high redshift, ALMA can only observe higher excitation lines, which are less affected. At $z>6$, only $\mathrm{CO}(6-5)\left(T_{\mathrm{e}}=116.16 \mathrm{~K}\right)$ and higher excitations can be observed with ALMA. The relation would still be valid at lower redshift $(z \lesssim 5)$. However, the effect of the higher CMB temperature needs to be examined carefully at higher redshifts.

Although we did not find any sign of redshift evolution, we also note that it is expected that the gas fraction in a galaxy increases as a function of redshift. Therefore, it is important to repeat this test when a larger sample becomes available in the future.

\subsection{Estimating gravitational magnification using the $L_{\mathrm{CO}}^{\prime} / \mu$-FWHM relation}

If we assume the $L_{\mathrm{CO}}^{\prime} / \mu$-FWHM relation, Eq. (2) can be used to estimate $\mu$. We rewrite the equation as

$\mu=L_{\mathrm{CO}}^{\prime} \times(F W H M / 1.3)^{-4.1}$.

The units for $L_{\mathrm{CO}}^{\prime}$ and FWHM are $\mathrm{K} \mathrm{Km} \mathrm{s}^{-1} \mathrm{pc}^{2}$ and $\mathrm{Km} \mathrm{s}^{-1}$, respectively. The equation can be useful when, for example, a sub-mm galaxy is detected behind a galaxy cluster and subject to gravitational magnification, but the detailed lens model is not available. Using Eq. (3), one can estimate $\mu$, hence intrinsic luminosities of the sub-mm galaxy.

\section{Discussion}

\subsection{A possible cosmological application}

If the correlation is independent of redshift, the luminosity distance to $\mathrm{CO}$-emitting galaxies can in principle be measured by estimating $L_{\mathrm{CO}}^{\prime}$ from FWHM. Such a distance measure would be useful because already now, galaxies at $z \sim 6$ have been detected in CO with ALMA (Riechers et al. 2013; Wang et al. 2013). This method can be used up to such a high redshift, where it is difficult for other distance measures to reach. Here we assess whether such an approach could be feasible.

In Figs. 1 and 2, $L_{\mathrm{CO}}^{\prime}$ is computed with a cosmology; $\left(\Omega_{\mathrm{M}}, \Omega_{\Lambda}, h\right)=(0.3,0.7,0.7)$. Therefore, we need to calibrate the correlation by using CO-detected galaxies with a measured distance from another method. This, however, is difficult because $\mathrm{CO}$ detected galaxies are mostly at very high redshifts where other distance measures cannot easily reach.

There are a number of ways to calibrate the distances, but here we use a method often used to calibrate gamma ray bursts. We use an empirical formula for the luminosity distance as a function of redshift based on 192 Type-Ia supernovae at $0.168<$ $z<1.755\left(D_{\mathrm{L}} / 10^{27} \mathrm{~cm}=6.96 z^{1.79}+14.79 z^{1.02}\right.$; Eq. (1) of Tsutsui et al. 2009). This empirical formula does not assume any cosmology, but just that the Type-Ia supernovae are standard candles. We use the formula to assign luminosity distances to our sub-mm galaxies at $z \leq 3$, and thus to calibrate our $L_{\mathrm{CO}^{-}}{ }^{-}$ FWHM correlation. Then, we apply the $L_{\mathrm{CO}}^{\prime}-\mathrm{FWHM}$ correlation to measure luminosity distances to the $z>3$ sub-mm galaxies.

The results are shown in Fig. 3. Along with the data points, we plot three different cosmological models with $\left(\Omega_{\mathrm{M}}, \Omega_{\Lambda}\right)=$ $(0.3,0.7),(0.3,0.0)$, and $(1.0,0.0)$. At this stage, it is clear that the scatter is more than an order of magnitude larger than needed to obtain meaningful constraints on the cosmological parameters. 


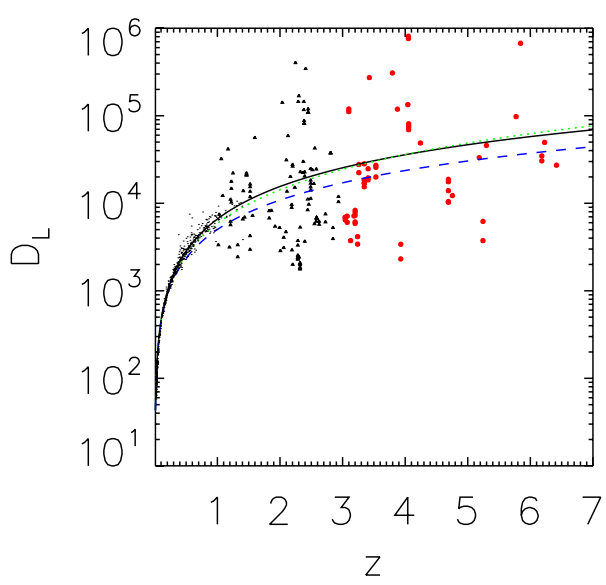

Fig. 3. Luminosity distance in Mpc against $z$. The red circles and black triangles are our $\mathrm{CO}$ samples separated at $z=3$. The black dots are 192 supernovae used to calibrate our low- $z$ sample. The black solid, green dotted, and blue-dashed lines are $\left(\Omega_{M}, \Omega_{\Lambda}\right)=(0.3,0.7),(0.3,0.0)$, and $(1.0,0.0)$, respectively.

\subsection{Sources of scatter in the relation, and prospects for reducing it}

The scatter around the $L_{\mathrm{CO}}^{\prime}-\mathrm{FWHM}$ correlation $(43 \%)$ is consistent with what is expected from randomly oriented disks, so if most sub-mm galaxies are rotating spiral-like galaxies, then the largest uncertainty is likely to come from the unknown inclinations. With the full operational array, ALMA's spatial resolution will be as good as 0.037 at $110 \mathrm{GHz}$ (for $\mathrm{CO}$ observation) in the extended configuration. At $z \sim 6$, this corresponds to $0.2 \mathrm{kpc}$, which is sufficient to resolve sub-mm galaxies, which typically extend over several kpc (Wang et al. 2013; De Breuck et al. 2014). With such observations, it will also be possible to exclude mergers and pressure-supported galaxies, which may be causing some of the extreme outliers from the relation, and to select samples of rotationally dominated galaxies. Furthermore, because the inclination is a random effect and its distribution is known (with $\langle\sin i\rangle \sim 0.79$ and $1 \sigma \sim 0.22$ ), one can statistically correct for the effect. For the Tully-Fisher relation, for example, it has been shown that even when no information on the inclination is available, by using maximum likelihood estimation, the true relation can be recovered with only a 1.5 times larger statistical error than when inclinations are known with zero uncertainty (Obreschkow \& Meyer 2013). As a reference, using the Tully-Fisher relation, Sakai et al. (2000) obtained $H_{0}=71 \pm 4$ (random) \pm 7 (systematic), i.e., $6 \%$ and $10 \%$ of the statistical and systematic errors, respectively.

Another possible source of scatter is calibration errors, because even state-of-the art sub-mm telescopes can only be calibrated to $10 \%$ at best for individual observations. Such errors are also expected to improve drastically as large, homogeneous statistical samples become available from ALMA, because the error on the median reduces with $\sqrt{N}$. In a sample of 10000 sub-mm galaxies, for example, the statistical error on the median will reduce to $1 \%$. This assumes the calibration error is random. If there is a systematic calibration error we are unaware of, this error will be added to the distance measurement.

Another possible source of scatter in the relation that will be decreased in future ALMA surveys, is conversions between CO transitions. In such samples, it will be possible to study galaxies in the same transition as a function of redshift or as subsets of galaxies with well-constrained transition conversions. Once the observational and systematic errors have been lowered enough to measure the intrinsic scatter of the relation, it will be necessary to assess its possible cosmological applications. At the moment, it is impossible to tell.

Acknowledgements. We greatly appreciate the anonymous referee, whose insightful comments helped in improving the paper significantly. We acknowledge the support by the Ministry of Science and Technology of Taiwan through grant NSC 103-2112-M-007-002-MY3. The Dark Cosmology Centre is funded by the Danish National Research Foundation.

\section{References}

Bothwell, M. S., Smail, I., Chapman, S. C., et al. 2013, MNRAS, 429, 3047 Carilli, C. L., \& Walter, F. 2013, ARA\&A, 51, 105

De Breuck, C., Williams, R. J., Swinbank, M., et al. 2014, A\&A, 565, A59

Fu, H., Cooray, A., Feruglio, C., et al. 2013, Nature, 498, 338

Harris, A. I., Baker, A. J., Frayer, D. T., et al. 2012, ApJ, 752, 152

Obreschkow, D., \& Meyer, M. 2013, ApJ, 777, 140

Riechers, D. A., Bradford, C. M., Clements, D. L., et al. 2013, Nature, 496, 329

Sakai, S., Mould, J. R., Hughes, S. M. G., et al. 2000, ApJ, 529, 698

Tsutsui, R., Nakamura, T., Yonetoku, D., et al. 2009, J. Cosmol. Astropart. Phys., 8,15

Tully, R. B., \& Fisher, J. R. 1977, A\&A, 54, 661

Wang, R., Wagg, J., Carilli, C. L., et al. 2013, ApJ, 773, 44 